Recepción: 09 / 03 / 2018

Aceptación: 28 / 05 / 2018

Publicación: 05 / 06 / 2018

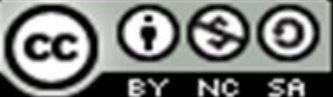

Ciencias sociales y políticas

Artículo de Investigación

\title{
Inclusión de las personas sexodiversas en la educación pública universitaria
}

\section{Inclusion of sexediverses in public university education}

\author{
A gestão humana desde a perspectiva do enfoque sistémico
}

\author{
Yadira V. Kure-Mejia ${ }^{\mathrm{I}}$ \\ yvkure@espe.edu.ec \\ Dustin D. Sarabia-Porras II \\ ddsarabia@espe.edu.ec \\ Nilton A. Guayta-Toapanta ${ }^{\text {III }}$ \\ alexeins_69@hotmail.com
}

Correspondencia: yvkure@espe.edu.ec

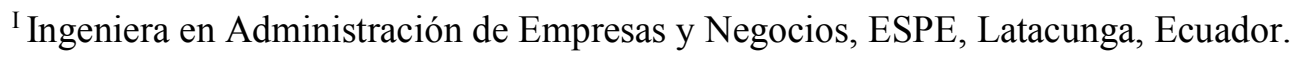

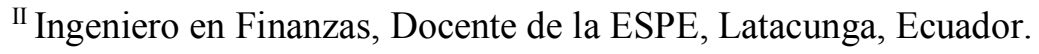

III Ingeniero Comercial, ESPE, Latacunga, Ecuador.
} 


\section{Resumen}

La inclusión social en las universidades es un tema reciente en América Latina. En la región son tímidas las propuestas de políticas públicas de inclusión de grupos sociales tradicionalmente excluidos en las instituciones de educación superior. La mayoría de estas propuestas atienden más que todo a la diversidad cultural y han pretendido incluir e integrar a la dinámica de los estudios universitarios a las personas de las etnias indígenas y pueblos afrodescendientes. y se ha dejado en un lugar marginal la inclusión de las personas sexodiversas a la universidad, sus vivencias en su tránsito por estas instituciones y, aún menos, su integración al campo laboral una vez graduados. Este estudio de tipo descriptivo que se complementará con un estudio de campo tomándose los datos directamente de la realidad por medio de la técnica de la encuesta a través de una escala lickert. Se halló que, aunque las personas sexodiversas tienen posibilidad de ingresar a la universidad, una vez adentro deben enfrentarse a discriminación social, sobre todo, en lo referente al respeto de sus derechos y al libre desarrollo de sus capacidades, este tipo de rechazo está vehiculado por violencia simbólica e invisibilización de su ser alterno a la heteronorma, sin embargo, hay una actitud resiliente que supera estos obstáculos e impide que se afecte el rendimiento académico de los estudiantes sexodiversos en el desempeño de su carrera universitaria.

Palabras clave: inclusión social; personas sexodiversas; educación superior; violencia contra la comunidad sexodiversa.

\section{Abstract}

Social inclusion in universities is a recent issue in Latin America. In the region, the proposals for public policies of inclusion of social groups traditionally excluded in institutions of higher education are timid. Most of these proposals focus more on cultural diversity and have sought to include and integrate the dynamics of university studies into people of indigenous and Afrodescendant peoples. and it has left in a marginal place the inclusion of sexediverse people to the university, their experiences in their transit through these institutions and, even less, their integration into the labor field once graduated. This descriptive study will be complemented with a field study taking the data directly from reality through the technique of the survey through a lickert scale. It was found that, although sexediverse people have the possibility of entering university, once inside they must face social discrimination, above all, with respect to the respect 
of their rights and the free development of their abilities, this type of rejection is conveyed for symbolic violence and the invisibility of its being to the heteronorm, however, there is a resilient attitude that overcomes these obstacles and prevents the academic performance of the different sexes students from being affected in the performance of their university career.

Keywords: social inclusion; sexless people; higher education; violence against the sexediversa community.

\section{Resumo}

A inclusão social nas universidades é um tema da América Latina. En la región soniges propuestas de policies públicas de inclusión de grupos sociales tradicionalmente excluidos en las instituciones de educación superior. A mayoría de these propuestas atienden más que todo a diversidade cultural e han pretendido inclui e integrar a dinamica dos estudantes universitários às pessoas das etnias indígenas e pueblos afrodescendientes. Em primeiro lugar, a maioria dos estudantes universitários tem a mesma universidad, sus vivencias en su tránsito por estas instituigóes y, aún menos, su integación al campo laboral una vez graduados. Este estudo descritivo se complementa com um estudio de campo para incluir os dados diretamente da realidade por medio da técnica da encurtar uma escala de uma escala lickert. Se você é um homem que gosta de ser sexodiversas, tem facilidade de se integrar na universidade, uma vez que adentro competir com uma discriminação social, sobre todo, e sobre o seu aliado aos seus débitos e outros resultados de seus esforços, este tipo de trabalho está por violencia simbólica e invisibilización de su alternate à heteronorma, is to be afetive the rendimiento académico de los estudiantes sexodiversos en el desempeño de su carrera universitaria.

Palavras chave: inclusão social; personas sexodiversas; educação superior; violencia contra la comunidad sexodiversa.

\section{Introducción}

El ser humano es un ser multidimensional, complejo e integral, cuyas principales dimensiones son la biológica, la intelectiva, la ética-moral y la volitiva-social o relacional, sin embargo, el ser humano también es un ser histórico, un ser en relación, un ser situado y entre, otros, es también un 
ser sexuado (Hurtado, 2006). Sin embargo, esta última característica, dentro de la sociedad patriarcal moderna se ha intentado omitir o invisibilizar desde resortes religiosos, que aún hoy se sigue asumiendo como tabú.

El tema de los derechos sexuales se considera de tercera generación, y aún hoy es un tema novedoso y desconocido por muchos. A este respecto es preciso destacar:

"Los derechos sexuales promueven la libertad de decidir y ejercer plenamente la sexualidad. Todas las personas somos intrínsecamente sexuales, y el desarrollo sexual evoluciona durante la infancia y la adolescencia, manifestándose durante el resto de las etapas de la vida". (Araujo-Cuauro, 2018, pág. 132)

Pero dentro de la sociedad moderna patriarcal en la cual vivimos, la sexualidad viene determinada por la construcción social de género que estima que solo hay una polaridad hombre-mujer heterosexuales y que todo lo que esté fuera de ella es susceptible de discriminación y omisión (Báez, 2016). De acuerdo con González y Smith (2014):

"Los roles de género son construcciones socio históricas sobre el comportamiento masculino y femenino basados en el sexo. Las marcas de género son troqueles decisivos en la construcción de los estereotipos y por ende de las desigualdades. Son ordenadores sociales que devienen en relaciones de poder y de subordinación”. (pág. 283)

Dentro de este esquema jerárquico el hombre heterosexual queda en la cúspide del poder social, mientras la mujer y las personas de la comunidad sexodiversa quedan en un lugar subalterno en la sociedad, situación que los lleva a luchar por igualdad de oportunidades y equidad social. Aquí es preciso definir que por género sexo diverso se busca reconocer:

"La existencia de una multiplicidad de vidas en la sociedad y una variedad de orientaciones, expresiones e identidades que contienen cargas afectivas, de género y sexo entre las personas como lo son el grupo de personas; Lesbianas, Gays, Bisexuales, Trans e intersex (LGBTI)”. (AraujoCuauro, 2018, pág. 133)

En este contexto, la homofobia, la lesbofobia, bifobia y transfobia generan violencia contra las personas del espectro LGBTI, generalmente cargada de chistes, bromas, ofensas, insultos y hostigamiento, algunas veces considerados como giros de humor socialmente aceptables, pero en 
realidad son formas sutiles y efectivas de violencia simbólica (Bastidas \& Torrealba, 2014). Este hecho no es nuevo, y se traslada a todos los espacios sociales, incluyendo a la universidad:

"La historia de la humanidad está cargada de pasajes sobre el maltrato a los individuos producto a su orientación sexual como lo es la comunidad de personas sexo-genero diversidad, término que reconoce la existencia de una multiplicidad de vidas en la sociedad y una variedad de orientaciones, expresiones e identidades que contienen cargas afectivas, de género y sexuales de y entre las personas lesbianas, gais, bisexuales, trans e intersex”. (Araujo-Cuauro, 2018, pág. 131)

Las mujeres, y en especial las personas del grupo sexo-diverso, se han visto tradicionalmente excluidas del sistema educativo, especialmente de la educación universitaria, pero en décadas recientes la mujer ha logrado acceder a las universidades, pero no así las personas del grupo LGBTI, que si bien acceden, usualmente deben esconder sus orientaciones sexuales o limitar sus expresiones corporales o modos de vida que los puedan identificar como pertenecientes a este grupo, en este caso, la personas trans e intersexuales, prácticamente se ven excluidas de la educación superior, máxime, en los países de América Latina.

Las políticas y programas de inclusión social suelen atender a la diversidad cultural y étnica, a la integración de sectores sociales menos favorecidos dentro del concepto de clase social, pero son pocos o nulos los intentos por estudiar y activar la inclusión, integración y acceso a la educación universitaria de todo el espectro sexo-diverso, este grupo es excluido en el discurso crítico sobre igualdad y acceso a la educación superior en América Latina que realizan Chan, García y Zapata (2013):

"La experiencia nos muestra que es posible encontrar evidencia sobre programas y políticas de acceso que han ido más allá de definiciones abstractas, mostrando resultados positivos entre colectivos usualmente excluidos como las mujeres, los afrodescendientes y los indígenas”. (pág. 131)

La educación, incluyendo a la universitaria se concibe como democrática, igualitaria y universal, donde se supone se debe promover la ciudadanía, la equidad y la inclusión social, para el debate de ideas (González \& Smith, 2014), pero según el juicio de Chan, García y Zapata (2013), estas son ideas abstractas que pocas veces en América Latina se traducen en instrumentalización de políticas y programas sociales de inclusión y equidad social, menos el acceso a la universidad. El 
ingreso a la universidad del espectro del grupo sexo-diverso es una tarea pendiente en los países de América Latina, empezando por el hecho que son pocos los estudios que den cuenta del ingreso y tratamiento igualitario de estas personas en el sistema de educación superior.

Bastidas-Terán, Arteaga-Rodríguez y Gómez-Martínez (2017) hallaron en una investigación en Valencia-Venezuela, que una mujer transgénero de 28 años, ni siquiera pensó en ingresar a la universidad para cumplir su sueño de ser médico, pues en ese país estas personas no tienen derecho a legalizar su identidad sexual auto-percibida, pero esta joven solucionó su situación integrándose a la religión del espiritismo donde no la excluyen por ser transgénero- formando parte de la corte chamarrera que es la encargada de la sanación física y espiritual.

Araujo-Cuauro (2018), estima que la identidad de género y la orientación sexual, son fundamentales en la construcción del proyecto de vida de las personas, por ende, una exclusión de este tipo puede afectar el desarrollo integral de las personas del género sexo-diverso. Un ejemplo vívido de esta situación fue reportado por Bastidas-Terán, Arteaga-Rodríguez y Gómez-Martínez (2017), quienes hallaron que un joven que se asumía como homosexual señaló que su vida productiva y laboral, la construyó a partir del estilismo y el modelaje, contextos laborales, donde según él, no había tanta discriminación por preferencias sexuales homosexuales, a diferencia de la mujer transexual que su paso por la universidad no estuvo en su proyecto de vida.

La universidad, como reflejo y antípoda de la sociedad, reproduce las discriminaciones sociales y culturales, donde se interceptan diferenciaciones multidimensionales, este hecho ha sido ignorado por los países de América Latina, de allí su poco avance en la inclusión y equidad en el acceso y convivencia universitaria:

"No obstante, el reconocimiento de las demandas y necesidades específicas de las poblaciones especiales en función de la diversidad étnica y cultural o por otras razones como la ubicación territorial, la discapacidad y las capacidades excepcionales- también han generado nuevas dimensiones de exclusión, en especial porque estas políticas y programas de inclusión social no asumen el carácter multidimensional de la exclusión”. (Chan, García, \& Zapata, 2013, pág. 132)

Este hecho es consistente por lo hallado por Castillo y Rivera (2015), quienes hicieron un estudio en el programa de Comunicación Social de la Universidad Centroamericana (Managua-Nicaragua), estas investigadoras, encontraron que efectivamente las discriminaciones en la convivencia 
universitaria es multidimensional e interceptada por múltiples categorizaciones donde las mujeres son blanco de violencia por razones de género, pero también por raza (color de piel), clase (vestimenta, equipos tecnológicos, educación, institución de procedencia), procedencia de provincia o sectores rurales subalternizados por quienes son de la capital; tensiones entre hombres sexodiversos y homofóbicos quienes también se vieron interceptados por discriminaciones raciales $\mathrm{y}$ de procedencia.

Esta situación no es nueva para las personas sexodiversas: "Sobre la violencia racial la CIDH, ha recibido información preocupante sobre los altos niveles de discriminación y violencia contra personas lesbianas, gais, bisexuales y trans (LGBTI descendencia africana) de y otras afectadas por la discriminación racial" (Araujo-Cuauro, 2018, pág. 147). Dentro de la universidad ya existen propuestas desde la perspectiva crítica feminista que abogan por la co-educación universitaria que tiene como finalidad: "propiciar el desarrollo integral de jóvenes en igualdad de derechos y oportunidades... En otras palabras, educar sin desconocer la unidad en la diversidad" (González \& Smith, 2014, pág. 279). Las autoras precitadas esgrimen que la co-educación es aprender participativamente, aprender a convivir con otros y educar en relación.

En consecuencia, para lograr esta co-educación, es fundamental primero analizar la inclusión de las personas sexodiversas a la educación pública universitaria, que representa el objetivo de la investigación presentada en este artículo.

\section{Metodología}

Esta investigación es de tipo descriptiva, porque enuncia las características de un evento o fenómeno (Palella \& Martins., 2006), en el caso de este estudio fue la inclusión de las personas sexodiversas en la vida universitaria. Se plantea un estudio de campo, tomando datos directamente de la realidad (Ramírez, 2007), por medio de la técnica de la encuesta a través de una escala lickert aplicada a estudiantes del grupo sexodiverso de una facultad de Ciencias Económicas y Sociales, una facultad de Derecho y una facultad de Humanidades y Educación. Dado que la población es desconocida, pues no hay registro estadístico de cuántas personas de la comunidad sexo-diversa hay en una institución de educación universitaria se encuestaron a 100 personas, mediante muestreo casuístico, es decir, se encuestaron las personas que accedieron a responder el instrumento. 
El instrumento tuvo una confiabilidad de 0,83 de acuerdo a la técnica alfa de crombach, lo que indica que dicha confiabilidad es fuerte, y fue validado por tres jueces expertos, un metodólogo, un sociólogo y un antropólogo. El procesamiento estadístico se realizó mediante estadística descriptiva representada en histogramas, organizadas por las dimensiones de la variable o evento, las cuales son: (a) igualdad de oportunidades, (b) respeto a sus derechos, (c) capacidad de expresión y desarrollo de habilidades.

\section{Resultados}

A continuación, se presentan los resultados por dimensiones de la variable:

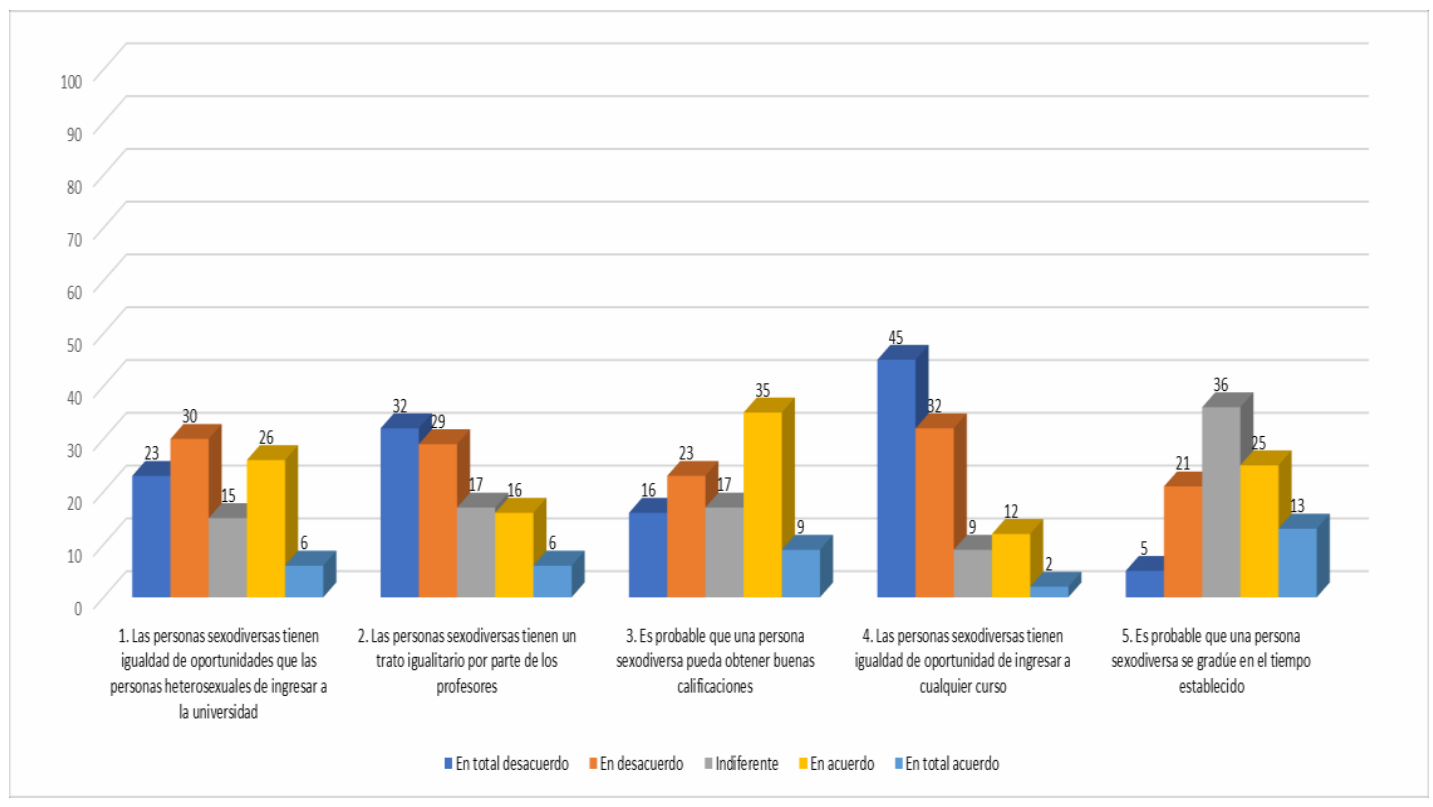

Gráfico 1. Dimensión 1: Igualdad de oportunidades

Fuente: Elaboración propia

En cuanto a la dimensión igualdad de oportunidades, el 45\% de los encuestados aseguró dijo estar en total desacuerdo con que la afirmación que los estudiantes sexodiversos tengan igual oportunidad de ingresar a cualquier curso, es decir, hay unidades curriculares donde se sienten más rechazados o excluidos que en otras, el 32\% señaló estar en desacuerdo con esta aseveración. Por otro lado, el 35\% de la muestra dijo estar de acuerdo con la afirmación que los estudiantes sexodiversos pueden obtener buenas calificaciones, esto fue contradicho por el $23 \%$ de los entrevistados quienes estuvieron en desacuerdo. 
Otro indicador que llama la atención, es el referido al enunciado que sentencia que las personas sexodiversas tienen un trato igualitario por parte de los profesores, el $32 \%$ dijo estar en total desacuerdo con esa afirmación, mientras un 29\% señaló estar en desacuerdo. Hay indicios que las personas sexodiversas son discriminadas por sus profesores. En otro orden de ideas, el 30\% indicó estar en desacuerdo con la afirmación que las personas sexodiversas tienen igual oportunidad de ingreso a las universidades, el 23\% dijo estar en total desacuerdo. E1 36\% precisó tener una posición indiferente frente a la afirmación que una persona sexodiversa tenga la oportunidad y posibilidad de graduarse en el tiempo estipulado, el 25\% indicó estar en acuerdo con esa aseveración. Al parecer, las discriminaciones no afectan sustancialmente el rendimiento académico de los estudiantes sexodiversos.

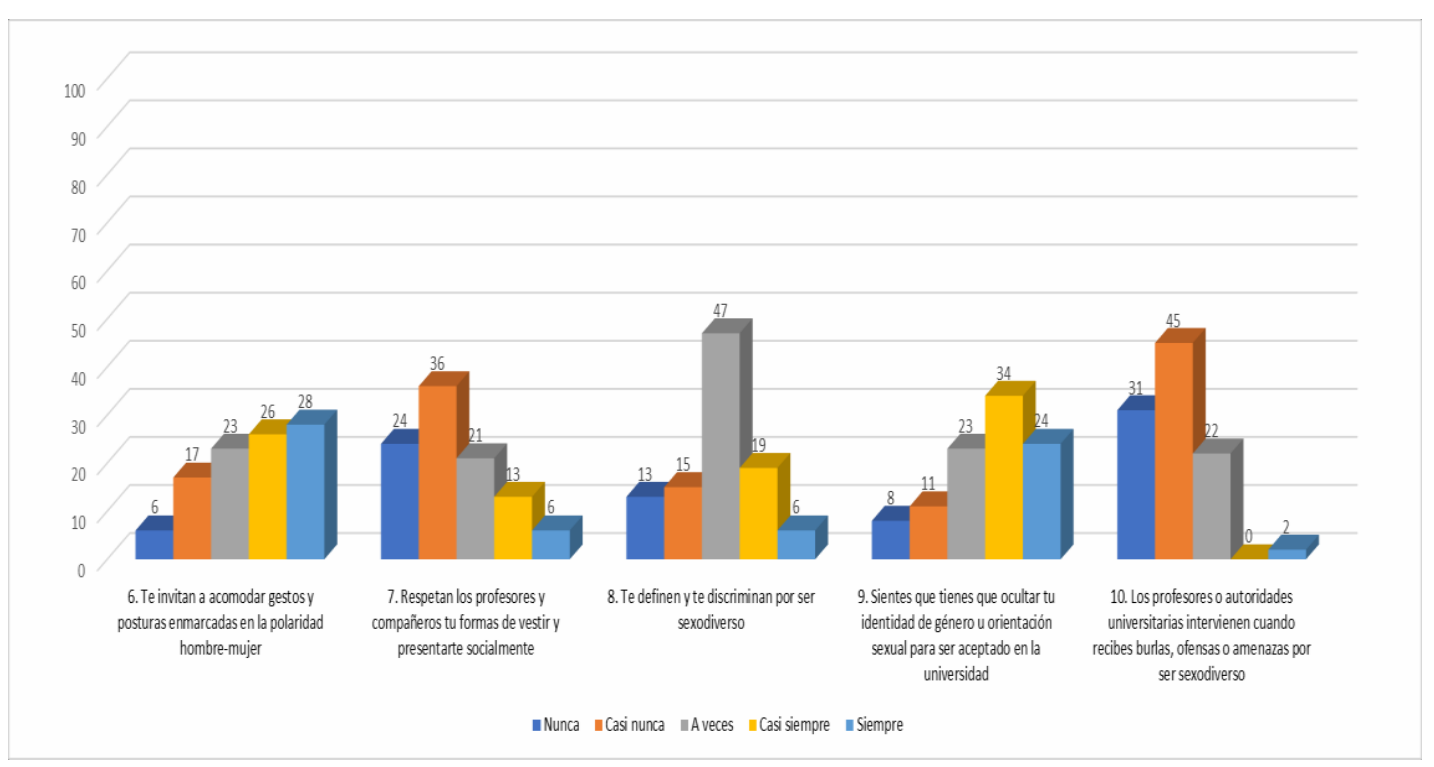

Gráfico 2. Dimensión 2: Respeto a sus derechos

Fuente: Elaboración propia

En la dimensión respeto a sus derechos, el $47 \%$ de los encuestado indicó que a veces lo definen y lo discriminan por ser sexodiverso, el 19\% precisó que esta situación ocurre casi siempre. Los derechos de los estudiantes universitario sexodiversos se ven afectados porque según el $45 \%$ de los encuestados dijeron que casi nunca las autoridades de su casa de estudios intervienen cuando reciben burlas, ofensas o a, el 31\% refiere que esto nunca sucede, lo que denota un ambiente hostil y poco inclusivo. 
Lo anterior tiene relación con el hallazgo de que el $36 \%$ de los consultados afirman que casi nunca los profesores y compañeros de estudios respetan sus formas de vestir y presentarse socialmente, el $24 \%$ indica que tal hecho nunca tiene lugar en su universidad. Como consecuencia de estas situaciones, el 34\% estima que casi siempre debe ocultar, simular o velar su identidad de género o su orientación sexual para no sufrir rechazo o discriminación, esto fue corroborado por el $24 \%$ que indicó que siempre se ve obligado a ello.

Finalmente, un $28 \%$ precisó que siempre se ve forzado o presionado a corregir modales y posturas de acuerdo a los patrones de género socialmente aceptados, el 26\% indicó que vive esta situación casi siempre, y el $23 \%$ señala que a veces recibe este mandato social en su casa de estudios. Sin duda hay discriminación y poco respeto a los estudiantes sexodiversos en la universidad en estudio, al limitarle su libertad para expresarse como personas.

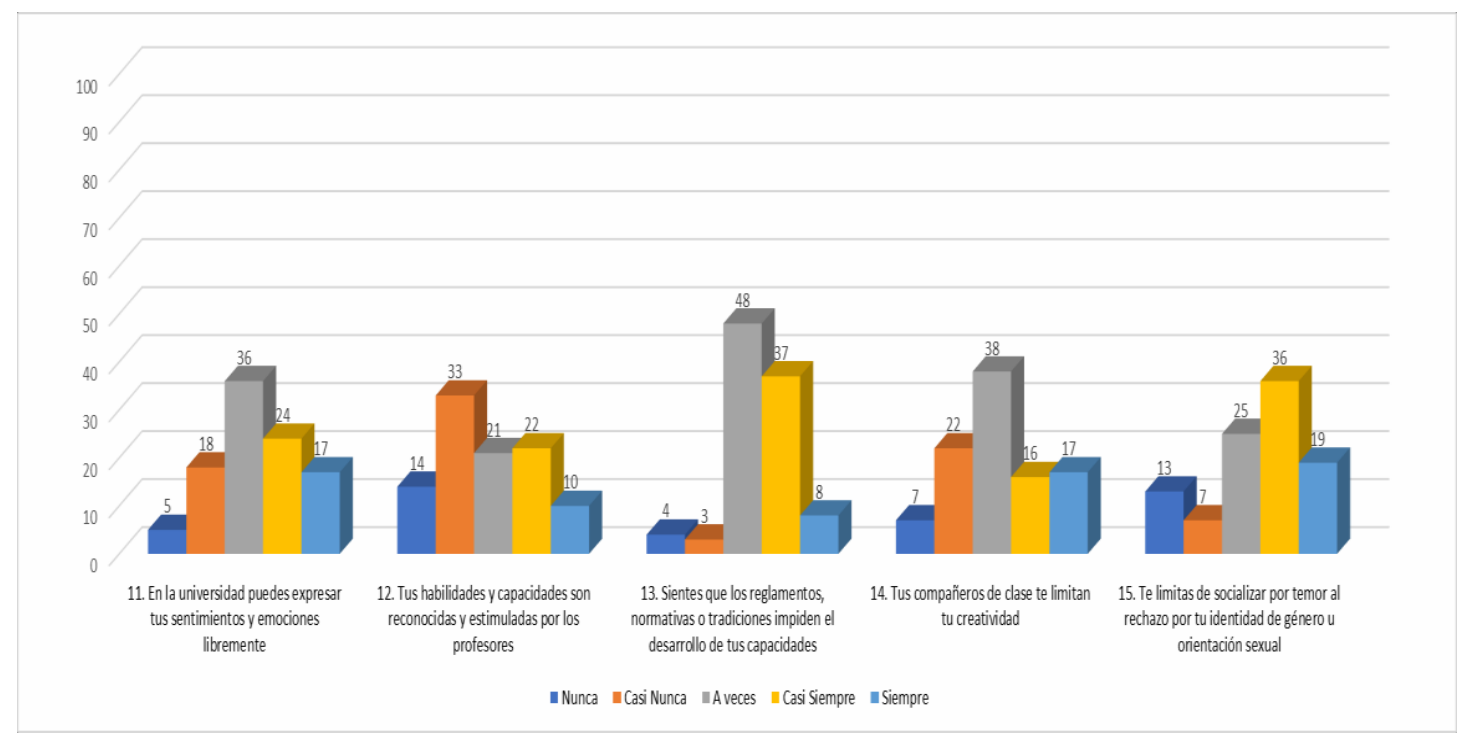

Gráfico 3. Dimensión 3: Capacidad de expresión y desarrollo de habilidades

Fuente: Elaboración propia

En lo relativo a la dimensión capacidad de expresión y desarrollo de sus habilidades el 48\% indica que a veces sienten que los reglamentos, normativas o tradiciones de la comunidad universitaria bloquean el desarrollo de sus capacidades, el 37\% precisa que esto ocurre casi siempre. El 38\% dice que esos bloqueos o limitaciones a su creatividad a veces viene por parte de sus compañeros, pero el $27 \%$ dice que esto casi nunca sucede. 
En sintonía con la dimensión anterior, el 36\% de los encuestados afirman que casi siempre se ve limitado a socializar en su contexto universitario por temor al rechazo por su identidad de género u orientación sexual, el $25 \%$ señala que a veces pasa por esa experiencia. Por su parte, el $36 \%$ precisa que a veces puede expresar sus emociones y sentimientos libremente, mientras el $33 \%$ dice que casi nunca los profesores reconocen el valor de sus aportes y propuestas.

\section{Conclusiones}

De acuerdo a este estudio, las personas sexodiversas en las universidades latinoamericanas aún no están incluidas socialmente, pues hay cursos donde no tienen las mismas oportunidades de ingreso, tampoco tienen un trato igualitario e inclusivo por parte de los profesores, hay divergencias en las percepciones en torno a las mismas oportunidades de ingreso a los estudios superiores por parte de las personas de género sexo-diverso, se observó que en lo atinente a rendimiento académico los estudiantes del grupo LGBTI pueden lograr un buen rendimiento académico referido tanto a calificaciones como a graduarse en el tiempo planificado. Faltaría revisar si esa aparente igualdad no tiene detrás un esfuerzo extra en comparación con sus compañeros heterosexuales o que aparentan serlo.

Los respetos a los derechos de la comunidad sexodiversa en la universidad parecen no ser la norma de inclusión social. La mayoría de los estudiantes del grupo LGBTI se sienten discriminados por su identidad de género u orientación sexual, esto se confirma porque, según lo hallado en este estudio, es común que las autoridades universitarias no intervengan en casos de discriminación o violencia contra las personas sexodiversas, menos tratan de mediar en estas situaciones, asunto que también es considerado agresión por negligencia.

Otra forma de vulneración de los derechos de las personas del grupo LGBTI en la universidad en estudio, fue el hecho que sienten irrespeto por su forma de ser y expresarse con modales, vestimenta o modos de presentarse socialmente, razón por la cual, sienten que los invitan a corregir esas actitudes consideradas fuera de la heteronorma, o bien a ocultar, simular o velar su identidad de género u orientación sexual.

En cuanto a la capacidad de desarrollar sus habilidades, estas se ven bloqueadas y limitadas por reglamentos, normativas y tradiciones de la comunidad universitaria, esto se instrumentaliza por medio del poco o nulo reconocimiento de su creatividad y potencialidad por parte de sus profesores 
y compañeros. Todo este clima hostil y poco inclusivo hace que los estudiantes sexodiversos se autocensuren y no logren socializar sin complejos en su sitio de estudios, por lo tanto, solo a veces pueden expresar libremente sus sentimientos y emociones.

Estos resultados coinciden con Araujo-Cuauro (2018), quien establece que las leyes internacionales y nacionales con respecto a la igualdad de género e inclusión social de la comunidad sexodiversa se queda en lo enunciativo y no pasa al plano práctico. Así mismo es consistente con la crítica realizada por Chan, García y Zapata (2013) quienes estiman que la inclusión social y equidad en el acceso y permanencia de grupos tradicionalmente excluidos en las universidades latinoamericanas se hace desde preceptos abstractos y enunciativos, pero que no se traducen en prácticas reales en la vida cotidiana de las instituciones de estudios superiores.

Con Bastidas y Torrealba (2014), hay coincidencias en el sentido que, aunque haya oportunidades de ingreso a las universidades, las personas socialmente discriminadas reciben violencia simbólica por medio de bromas, chistes, ofensas e invisibilización de sus capacidades, tal como es el caso de la comunidad sexodiversa en su tránsito por la vida universitaria, todo esto las lleva a autocensurarse.

El hecho que a pesar de que existe un ambiente o clima organizacional de las universidades desfavorable a la inclusión de las personas sexodiversas, estos individuos son resilientes y consideran que su rendimiento académico no se ve afectado por las discriminaciones y omisiones de que son objeto, esta capacidad resolutiva y resiliente de las víctimas de invisibilización es consistente con los hallazgos de Bastidas-Terán, Arteaga-Rodríguez y Gómez-Martínez (2017) quienes reportaron que un joven homosexual y una mujer transgénero resolvieron creativamente seguir con sus proyectos de vida a pesar que la sociedad bloqueaba ese impulso negándoles desarrollarse en ciertos espacios "institucionales".

Restaría investigar como las discriminaciones en la universidad contra el grupo LGBTI se intercepta con otras discriminaciones por género, clase social, origen étnico, criterios raciales y lugar de procedencia, y otros como creencias religiosas, tal como lo hallaron Castillo y Rivera (2015) cuyo estudio se centró más en la discriminación a la mujer. Finalmente, habría que desarrollar la idea de co-educación universitaria de González y Smith (2014) pero ampliándola de la igualdad de género hombre-mujer a la inclusión de género sexo-diverso. 


\section{Referencias bibliográficas}

Araujo-Cuauro, J. (2018). La violencia contra las personas sexo-género diversos y tratamiento en el sistema jurídico venezolano. TELOS. Revista de Estudios Interdisciplinarios en Ciencias Sociales, 20(1), 129-158.

Báez, M. (2016). Desafíos de la comunidad sexodiversa en las políticas sociales. Una perspectiva de género necesaria. En F. Bastidas, Una mirada a la Venezuela otra. Bitácora inicial. ValenciaVenezuela: Grupo de Investigación Alteridad Latinoamericana y Caribeña-Universidad de Carabobo. Obtenido de http://mriuc.bc.uc.edu.ve/handle/123456789/4272

Bastidas, F., \& Torrealba, M. (2014). Definición y desarrollo del concepto "proceso de invisibilización" para el análisis social. Una aplicación preliminar a algunos casos de la sociedad venezolana. Espacio Abierto, 23(3), 515-533. Obtenido de http://www.redalyc.org/articulo.oa?id=12232258007

Bastidas-Terán, F., Arteaga-Rodríguez, A., \& Gómez-Martínez, J. (2017). Fundamentalismo religioso, género y procesos de invisibilización según la vivencia de algunas de sus víctimas en la Gran Valencia, Venezuela. Boletín Científico Sapiens Research, 7(1), 33-47. Obtenido de http://www.srg.com.co/bcsr/index.php/BCSR/article/view/120/107

Castillo, V., \& Rivera, M. (2015). ¿La universidad, un espacio inclusivo? Representaciones sociales sobre situaciones de discriminación entre estudiantes de comunicación social de la Universidad Centroamericana. Managua: Universidad Centroamericana. Obtenido de http://repositorio.uca.edu.ni/2633/1/UCANI3953.pdf

Chan, J., García, S., \& Zapata, M. (2013). Inclusión social y equidad en las Instituciones de Educación Superior de América Latina. ISEES(13), 129-146. Obtenido de DialnetInclusionSocialYEquidadEnLasInstitucionesDeEducaci-4421725.pdf

González, M., \& Smith, Y. (2014). Co-educación: Una vía para lograr construcción ciudadana desde los espacios universitarios. Reflexiones para el debate. Revista Ciencias de la Educación, 24(44), 277-292. Obtenido de www.researchgate.net/profile/Yamile_Delgado_De_Smith/publication/281280982_COEDUCATION_A_PATH_TO_ACHIEVE_THE_CONSTRUCTION_OF_CITIZENSHIP_FRO 


\section{M_THE_UNIVERSITY_CONTEXT_REFLEXIONS_FOR_DEBATE/links/55def58208ae45e82} $5 \mathrm{~d} 3 \mathrm{bde} 3 . \mathrm{pdf}$

Hurtado, J. (2006). Procesos grupales y psicología de la integración. Caracas-Bogotá: CieaSypalQuirón.

Palella, S., \& Martins., F. (2006). Metodología de la investigación cuantitativa. Caracas: FEDUPEL.

Ramírez, T. (2007). ¿Cómo hacer un proyecto de investigación? Caracas: Panapo. 